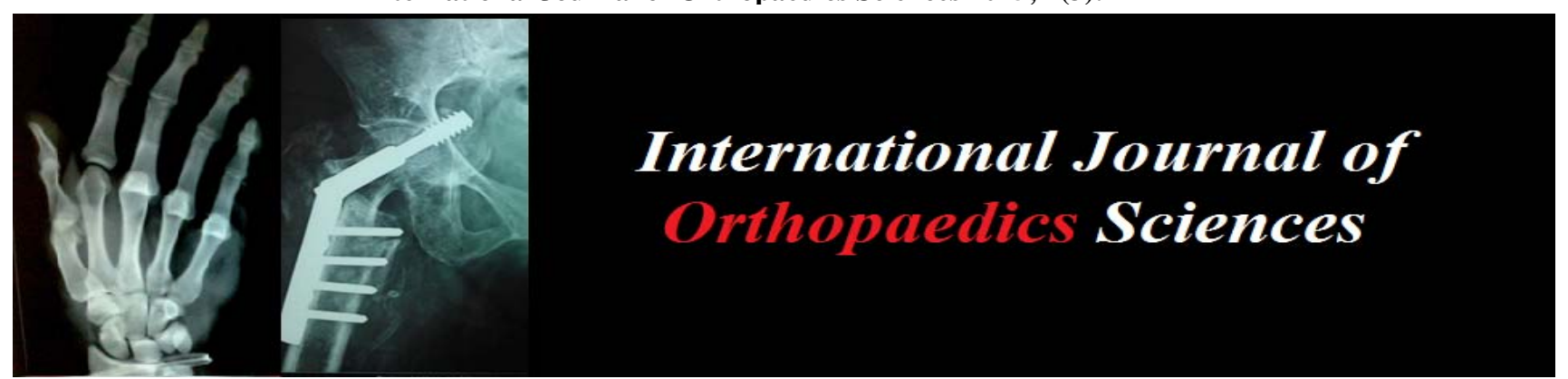

ISSN: 2395-1958

IJOS 2015; 1(3): 14-17

(C) 2015 IJOS

www.orthopaper.com

Received: 21-06-2015

Accepted: 22-07-2015

Praveen Kumar Pandey

Senior Resident Doctor,

Deptt. of Orthopaedics,

ESI-PGIMSR Model Hospital,

Basaidarapur, New Delhi, India.

Inder Pawar

Associate Professor,

Deptt. of Orthopaedics,

ESI-PGIMSR Model Hospital,

Basaidarapur, New Delhi, India.

Raaghav Rai Verma

Senior Resident Doctor,

Deptt. of Orthopaedics,

Dr RML Hospital,

New Delhi, India.
Correspondence

Praveen Kumar Pandey

Senior Resident Doctor,

Deptt. of Orthopaedics,

ESI-PGIMSR Model Hospital,

Basaidarapur, New Delhi, India.

\section{Case of tuberculosis acromioclavicular joint presenting as lytic lesion in lateral end clavicle: rare case report}

\author{
Praveen Kumar Pandey, Inder Pawar, Raaghav Rai Verma
}

\begin{abstract}
Background: Rise in the incidence of tuberculosis (TB) seen in recent years. Skeletal TB accounts for less than $10 \%$ of all cases of TB. Most of these cases are in the spine and hip. The acromio-clavicular (AC) joint is a very uncommon site of skeletal TB.

Case report: A 30 year old female developed pain in her right shoulder after sustaining trivial trauma. She was treated conservatively for six weeks, but patient complaints of increase in the intensity of pain with restricted movements. Radiographs repeated after six weeks showed a lytic lesion in the lateral end of clavicle with differential diagnosis of a solitary bone cyst or metastatic lesion. FNAC of the swelling done and MRI right shoulder advised. Histo-pathological report revealed evidence consistent with TB. The patient started on a multidrug anti-tubercular therapy (ATT) regimen with diagnosis of tuberculosis lateral end clavicle. MRI report after two weeks showed synovial hypertrophy and capsular distension with mild joint effusion in right AC joint. The diagnosis changed to tuberculosis of AC joint with continuation of the same treatment.

Conclusion: The rarity of the occurrence of TB at unusual sites, and the ability of tuberculosis to mimic other diseases, combined with a lack of awareness by the treating orthopaedist often leads to diagnostic delays. A high index of suspicion should be maintained in cases of swellings around the shoulder, even if they lack systemic features of tuberculosis.
\end{abstract}

Keywords: Tuberculosis; acromio-clavicular joint; lytic; clavicle; lateral end.

\section{Introduction}

There has been a rise in the incidence of Tuberculosis (TB) in recent years. This increase appears to be due to several factors, including development of resistance to commonly used chemotherapeutic substances, widespread immigration, HIV infection and illicit drug abuse. This resulted in a world-wide resurgence of tuberculosis as an important cause of osteo-articular infection ${ }^{[1]}$ and more and more cases are being reported with unusual features. Skeletal TB accounts for less than $10 \%$ of all cases of TB. Most of these cases are in the hip \& spine while involvement of the appendicular skeleton is unusual. The acromioclavicular (AC) joint is an uncommon site of skeletal tuberculosis, reportedly accounting for less than one percent of cases ${ }^{[2]}$. Conventional X-rays often fail to detect early skeletal disease in addition, the radiological picture is variable and the lytic bony lesions may easily be confused with osseous, inflammatory or neoplastic diseases, either primary or metastatic, resulting in a delayed diagnosis and delays in appropriate therapy. Very rare occurrence of TB AC joint and coincidental finding of tuberculosis acromio-clavicular joint in a patient with pain right shoulder after trivial trauma prompted us to report this unusual case.

\section{Case Report}

A 30 years/female developed pain in her right shoulder after sustaining trivial trauma, when she fell on the ground. She presented to orthopaedics emergency after a fall where shoulder x-rays done and diagnosed as soft tissue injury right shoulder with no associated bony injury. She was treated with anti-inflammatory medications and arm pouch sling for two weeks, but pain persisted in right shoulder. She came back to orthopaedics OPD for the same after two weeks where she was advised shoulder physiotherapy. Even after four weeks of intensive shoulder physiotherapy patient complaints of increase in the intensity of pain with restricted movement of right shoulder. Radiographs were repeated at this stage; they were interpreted by radiologist as well as treating orthopaedist as a lytic lesion in the lateral end of clavicle with periosteal reaction along the superior border (figure 1) with differential diagnosis of solitary bone cyst, 
metastatic lesion or skeletal TB in mind. Clinical examination of the shoulder revealed a soft tissue swelling (figure 2) and tenderness at the lateral end of clavicle with no discharging sinuses around, there was no evidence of cervical or axillary lymphadenopathy and the chest X-ray was essentially clear. She was advised to undergo FNAC of the swelling and MRI right shoulder with clavicle and AC joint.

Laboratory tests showed the complete blood count report within normal limits and ESR was $40 \mathrm{~mm} / \mathrm{hr}$ (Normal- less than 20mm/hour).

The FNAC report revealed evidence of epitheloid cell granulomas and necrosis consistent with tuberculosis (figure 3). The patient was started on a multidrug antitubercular therapy regimen(ATT) consisting of four drugs(Rifampicin $600 \mathrm{mg} /$ day, Isoniazid $300 \mathrm{mg} /$ day, Ethambutol $1000 \mathrm{mg} /$ day and Pyrazinamide $1500 \mathrm{mg} /$ day) with diagnosis of tuberculosis lateral end clavicle. MRI report ( figure 4) after two weeks of starting ATT showed well-defined oval soft tissue lesion measuring $9 * 23 * 14 \mathrm{~mm}$ in the subcutaneous plane of the right deltoid region communicating with the right acromio-clavicular joint and extend along the bursal surface of myotendinous part of supraspinatous muscle. The lesion is hyper-intense on T2 weighed image. Right AC joint showed synovial hypertrophy and capsular distension with mild joint effusion. The diagnosis changed to tuberculosis of AC joint with continuation of the same treatment. Pain and restriction of movements in the right shoulder improved within four weeks of starting anti-tubercular therapy. After six months of anti-tubercular therapy patient became completely asymptomatic with no restriction of movements in the right shoulder and the resolution of lesion seen on radiographs (figure 5). Patient was followed up regularly after completion of the course of anti-tubercular therapy.

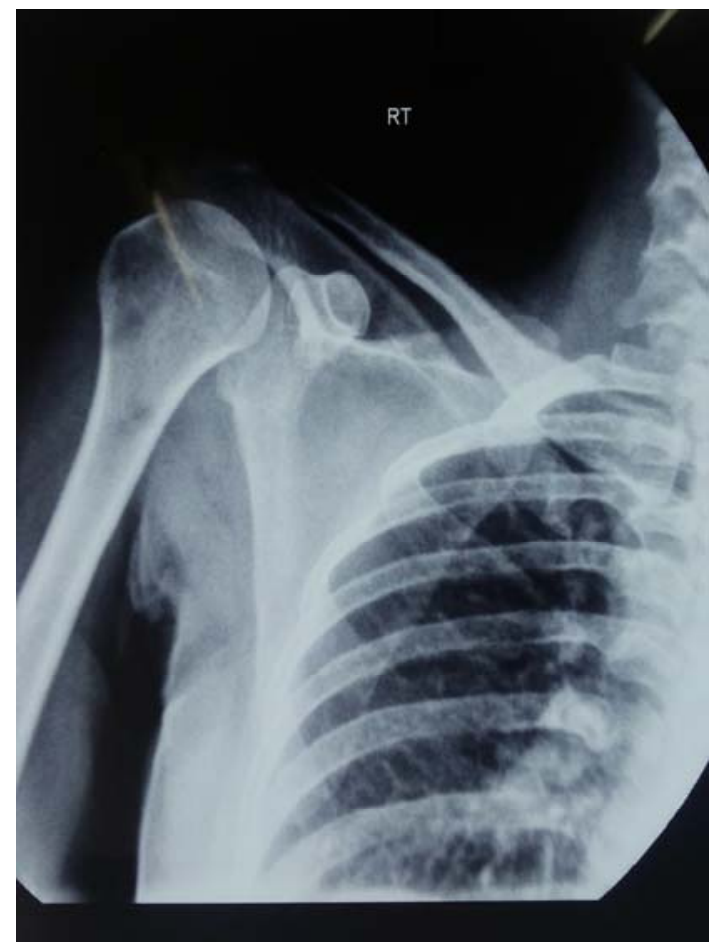

Fig 1: X-ray chest with right clavicle AP view showing lytic lesion in lateral end of right clavicle.

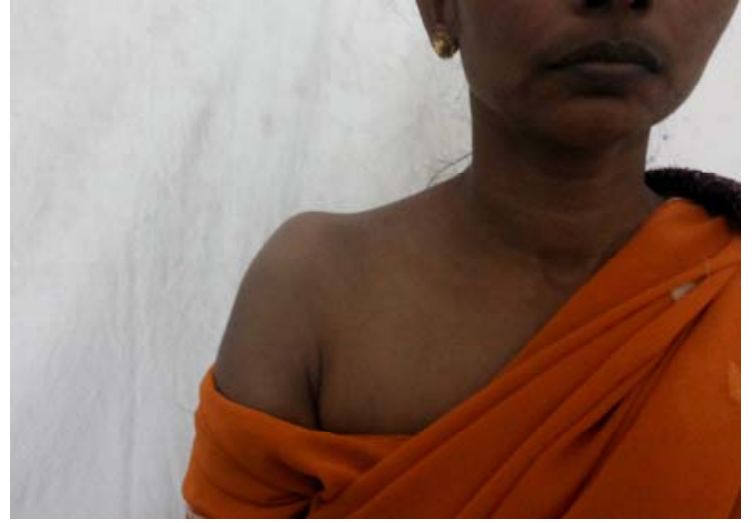

Fig 2: clinical photograph of the patient showing swelling over lateral end of clavicle region

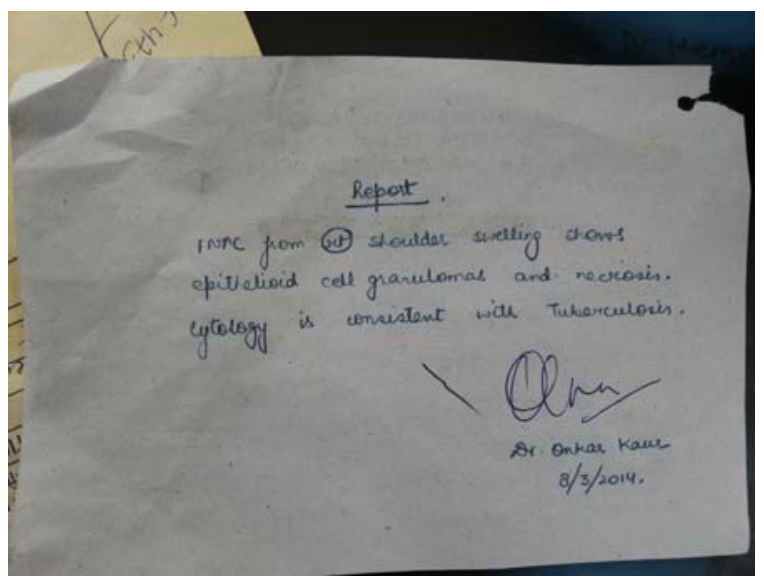

Fig 3: FNAC report of swelling right lateral end clavicle consistent with tuberculosis

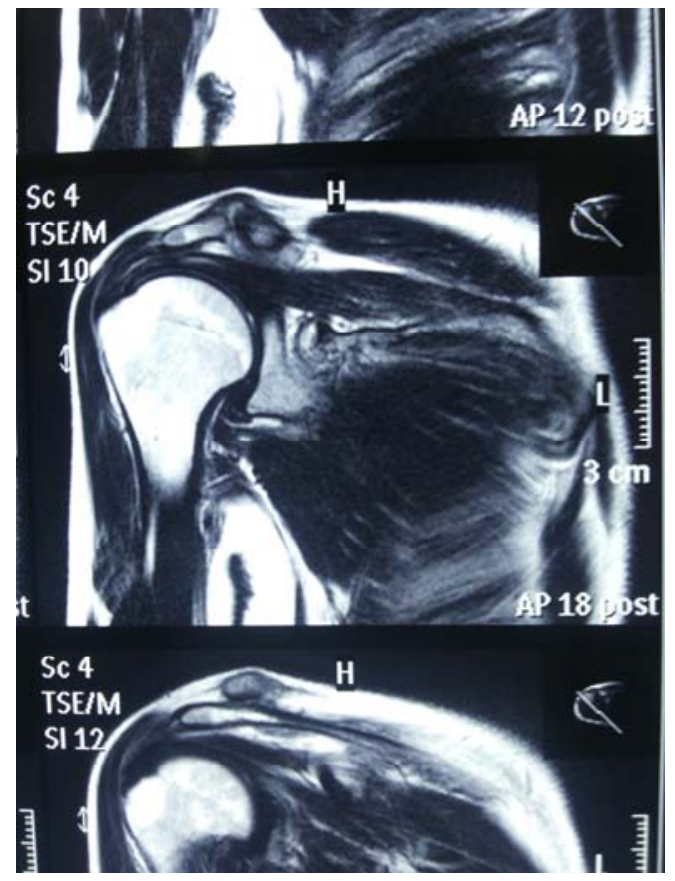

Fig 4: MRI right shoulder joint with clavicle coronal cuts showing pathology in right acromio-clavicular joint 


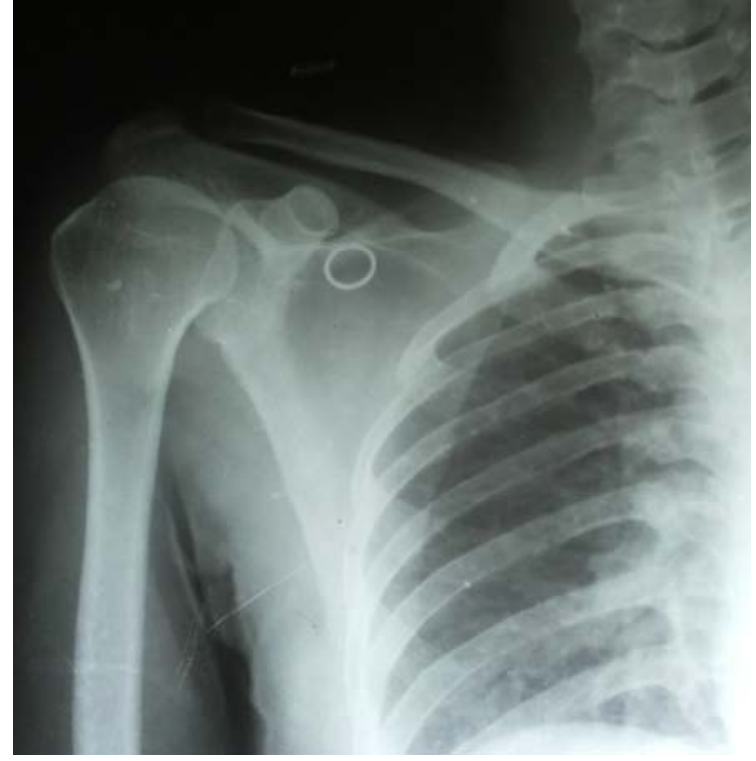

Fig 5: X-ray chest with right clavicle AP view after six months of ATT showing resolution of lytic lesion in lateral end clavicle

\section{Discussion}

Out of all TB cases, about $15 \%$ of them occur at extrapulmonary sites and about 3-5\% of them seen in osteo-articular system ${ }^{[9]}$. Most of osteo-articular TB cases occur in the hip \& spine. Involvement of the appendicular skeleton is unusual. With the increasing incidence of immune-compromised patients, a significantly older population, and the emergence of multidrug resistant strains, the problem of osteo-articular TB is becoming more prominent. Richter et al. reported an average delay of fifteen months between the beginning of symptoms and diagnosis ${ }^{[4]}$. This delay could be attributed to the slow, insi dious pathologic process ${ }^{[5,6]}$. Radiologically osteo-articular TB presented as a lesion showing osteoporosis, subchondral erosions, diminished joint space, reactive sclerosis and progressive joint destruction ${ }^{[7]}$. This is unfortunate that with the passage of time there is development of either a sinus or involvement of the adjacent joint which is worse than the purely osteomyelitis stage, in terms of superimposed infections or decreased joint motion after healing. The rarity of the occurrence of tuberculosis at unusual sites and the ability of tuberculosis to mimic other diseases, combined with a lack of suspicion by the treating orthopaedist, especially in communities where the disease is not routinely prevalent, often leads to diagnostic delays. This frequently poses diagnostic difficulties ${ }^{[2]}$, as the clinician may not keep the possibility of TB primarily in mind when disease occurs at unusual sites. Tubercular involvement of the AC joint is extremely rare ${ }^{[8], ~[9]}$ which prompt us to report our case of TB AC joint. A literature review revealed only three cases of TB AC joint reported in the last 60 years ${ }^{[10-12]}$, one case associated with miliary $\mathrm{TB}^{[10]}$, another case associated with scrofuloderma ${ }^{[12]}$. Recently mittal et al. reported two cases of acromioclavicular joint tuberculosis [13]. Tuberculosis of the AC joint may differ clinically and pathologically from tuberculosis of other joints and is difficult to diagnose in the early stages. Infection at this site (AC joint) has been reported to be more frequently of the destructive or proliferative type and may also be complicated by pathologic fracture ${ }^{[8,14]}$. Even the joint space reduction and the surrounding rarefaction, a feature typical of osteoarticular TB elsewhere in the body, is not so commonly observed at this site. Diagnostic problems are confounded by the fact that this site is more prone to the development of neoplasms as compared to infections ${ }^{[1}$,
15]. Osteo-articular TB is almost always secondary to a primary focus elsewhere in the body ${ }^{[15,16]}$, which may be dormant or active in state and a definite attempt should be made to screen the pulmonary, GIT and renal systems for evidence of disease. In our case, we didn't find any primary focus. The basis for an accurate diagnosis of TB is obtaining representative tissue from the focus or the isolation of Mycobacterium tuberculosis on culture which we get using FNAC method in our case. In our experience with such cases, as well as that of leading workers in this field ${ }^{[9,17]}$, there is a high incidence of false-negative culture reports. This is because of the reason that osteo-articular tuberculosis is a paucibacillary disease in comparison to the number of bacilli seen in pulmonary lesions. In most of the cases, however, the diagnosis has to be made by clinical features, concomitant pathology, histo-pathological evidence of granulomatous tissue, and a high index of suspicion ${ }^{[1,15]}$. In our case we found AC joint subluxation, absence of systemic manifestations, and a lytic lesion in metaphyseal location on radiographs which were certain peculiarities that confused the picture. The treatment of skeletal TB is medical, and surgical intervention is needed only for the purpose of obtaining tissue for diagnosis. Once the appropriate anti-TB therapy is started, the symptoms resolve within 6 to 8 weeks, and most discharging sinuses heal. We recommend a four-drug regimen for a minimum period of 3-4 months, and once the clinical features settle, the patient should be maintained on a two-drug regimen for an additional 9 to12 months.

In conclusion, we would like to emphasize the fact that a radiological picture of a lytic lesion in the lateral end of clavicle should include tuberculosis in its differential diagnosis. A high index of suspicion should be maintained in cases of gradually progressive swelling around the shoulder, even if they lack systemic features of tuberculosis. AFB culture is not essential for diagnosis and once a histopathology report confirms the diagnosis of tuberculosis, multidrug medical therapy for a prolonged period should be started.

\section{Compliance with ethical standards \\ Funding: not applicable.}

Conflict of interest: Author 1 to 3 declares that they have no conflict of interest.

Ethical approval: All procedures performed in our case report involving human participant were in accordance with the ethical standards of the institutional and/or national research committee and with the 1964 Helsinki declaration and its later amendments or comparable ethical standards.

Informed consent: Informed consent was obtained from the patient included in our study. Patient has given consent for the Case report to be published.

\section{References}

1. Abdelwahab IF, Kenan S, Hermann G. Atypical skeletal tuberculosis mimicking neoplasm Br. J Radiol. 1991; 64:551-555.

2. Gerscovich EO, Greenspan A. Osteomyelitis of the clavicle: Clinical, radiologic, and bacteriologic findings in ten patients. Skel. Radiol 1994; 23:205-210.

3. Rafiqi K, Yousri B, Arihi M. Unusual locations of osteoarticular tuberculosis in children: a report of 12 cases. Orthop TraumatolSurg Res, 2013, 347-51.

4. Richter R, Hahn H, Nubling W, Kohler G. Die Schulter gurel and Schultergelenktuberkulose. Z. Rheumatol 1985; 44(2):87-92. 
5. Bateman J. The Shoulder and Neck. WB Saunders Co. Philadelphia, 1975.

6. Tuli SM. Tuberculosis of the shoulder. Tuberculosis of the Skeletal System. 1st Ed. New Delhi. Jaypee Brothers Medical Publisher (P) Ltd, 1993.

7. Wolfgang GL. Tuberculous joint infection. Clin Orthop 1978; 136:157-63.

8. Rasool MN, Govender S. Infections of the clavicle in children. Clin. Orthop 1991; 265:178-182.

9. Tuli SM, Sinha GP. Skeletal tuberculosis unusual lesions. Indian J Orthop. 1969; 3:5-19.

10. Agathangelidis F, Boutsiadis A, Fouka E, Karataglis D. Concomitant Acromioclavicular and miliary tuberculosis. BMJ Case Rep 2013; 21:2013.

11. Taub SJ, Friedman IA, Dibble JB. Tuberculosis of the acromioclavicular jointwith subscapular bursitis and effusion Ill Med J. 1950; 98(6):357-60.

12. Tan WP, Tang MB, Tan HH. Scrofuloderma from the acromioclavicular joint presenting as a chronic ulcer in an immunocompetent host. Singapore Med J 2007; 48(9):2435.

13. Mittal R, Kandwal P, Rao SS, Kumar R. Acromioclavicular joint tuberculosis: apropos of two cases. Acta Orthop Traumatol Turc. 2014; 48(5):590-2.

14. Jensen SR. Tuberculous osteomyelitis of the clavicle. A report of five cases. Acta Chir. Scand 1959-1960; 118(Suppl.):327-330.

15. Fang JT, Huang CC, Liu HP. Apparent neoplasm of the clavicle of a dialysis patient, ultimately revealed as tuberculosis. Nephrol. Dial. Transplant 1996; 11:13801382.

16. Shannon B, Moore M, Houkom J, Waecker Jr NJ. Multifocal cystic tuberculosis of bone. J Bone Joint Surg. 1990; 72-A: 1089-1092.

17. Shrivastava KK, Garg LD, Kochar VL. Tuberculosis osteomyelitis of the clavicle. Acta Orthop. Scand 1974; 45:668-672. 